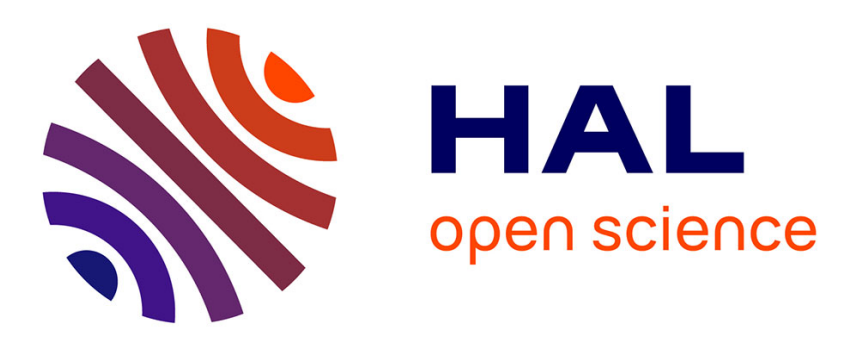

\title{
Non-smooth contact dynamics approach of cohesive materials
}

\author{
Michel Jean, Vincent Acary, Yann Monerie
}

\section{To cite this version:}

Michel Jean, Vincent Acary, Yann Monerie. Non-smooth contact dynamics approach of cohesive materials. Philosophical Transactions of the Royal Society of London Series A Mathematical and Physical Sciences (1934-1990), 2001, 359 (1789), pp.2497-2518. 10.1098/rsta.2001.0906 . inria-00423556

\section{HAL Id: inria-00423556 https://hal.inria.fr/inria-00423556}

Submitted on 3 May 2016

HAL is a multi-disciplinary open access archive for the deposit and dissemination of scientific research documents, whether they are published or not. The documents may come from teaching and research institutions in France or abroad, or from public or private research centers.
L'archive ouverte pluridisciplinaire HAL, est destinée au dépôt et à la diffusion de documents scientifiques de niveau recherche, publiés ou non, émanant des établissements d'enseignement et de recherche français ou étrangers, des laboratoires publics ou privés. 


\title{
Non-smooth contact dynamics approach of cohesive materials
}

\author{
Michel Jean, Vincent Acary and Yann Monerie \\ Laboratoire de Mécanique et d'Acoustique, CNRS-ESM2 Technopôle de Château-Gombert,
} 13451 Marseille, Cedex 20, France

The main features of the non-smooth contact dynamics (NSCD) method-the dynamical equation, the Signorini relation as a non-smooth modelling of unilateral contact, and the frictional Coulomb's law, treated with fully implicit algorithmsare briefly presented in this paper. By mere changes of variables, it appears that a large class of interface problems, including cohesive interface problems, may be solved using Signorini, Coulomb and standard NSCD algorithms. Emphasis is put on contact between deformable bodies. Examples illustrating numerical simulation are given for fibre-reinforced materials and for buildings made of blocks.

\section{Introduction}

Non-smooth mechanics embraces mechanical problems described by steep functions or functions with steep derivatives or non-differentiable functions. For instance, a heterogeneous elastic material, composed of stiff elastic inclusions imbedded into a soft elastic medium, is described by a local rigidity fourth-order tensor function which appears to be strongly discontinuous from a stiff domain to a soft domain. From the finite-element numerical point of view, solving the continuous medium equilibrium equation leads us to solve a linear problem (as far as small perturbations are concerned), involving the elasticity matrix, which happens to be ill conditioned. If the inclusions are nearly rigid bodies, or real rigid bodies, some special techniques are to be used, which belong to the field of non-smooth mechanics. One way of dealing with a rigid inclusion is to adopt, as in rigid bodies mechanics, a description with six degrees of freedom (only three in two-dimensional problems) accounting for translational and rotational motions. The points of the boundary separating the soft material and the rigid inclusion are subjected to the rigid velocity field of the inclusion. Though there might be some other ways of dealing with rigid inclusions, these considerations enhance the part played by boundary conditions, and lead us to consider more sophisticated boundary conditions, such as unilaterality, friction and cohesion. Actually, in this paper, emphasis is put on these kinds of boundary condition.

Another feature of non-smooth mechanics is the inclination to adopt behaviour laws, accounting roughly for the real behaviour, discarding the details, either because insufficient knowledge or data do not allow a meaningful description, or because it is decided that taking into account those details will not bring significant changes, and, consequently, these details should be ignored for the sake of simplicity. For instance, 

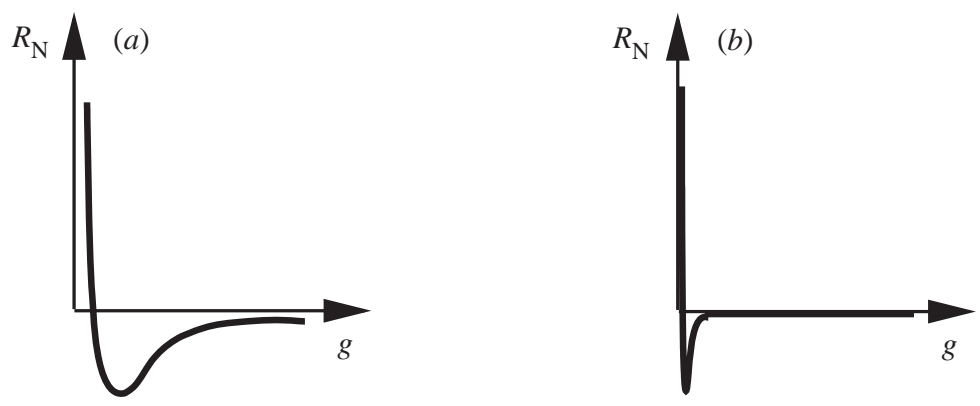

Figure 1. (a) Standard and (b) large-scale Lennard-Jones graph.
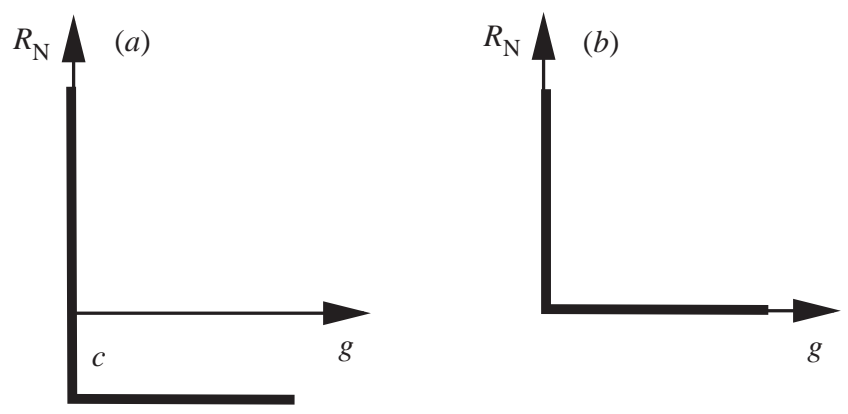

Figure 2. 'Non-smooth' cohesive law: (a) with cohesive status; (b) without cohesive status.

impenetrability is expressed by writing that the gap, the distance $g$ between some candidate point and the antagonist body boundary, is positive, $g \geqslant 0$. Together with the fact that the reaction force should have a positive normal component $R_{\mathrm{N}} \geqslant 0$, vanishing if the gap is strictly positive, $g R_{\mathrm{N}}=0$, one arrives at the so-called Signorini condition. Another way, going into details, is to consider that the bodies are rough, and that contact is set between elastic asperities scattered on some smooth reference boundaries surfaces. The gap $g$ should be understood as the distance between these reference surfaces, and might take negative values when asperities are deformed. The normal component of the reaction force might appear to depend linearly on the penetration distance, $R_{\mathrm{N}}=-k g$, where $k$ is some stiffness related to the elastic properties of the asperities. The normal reaction force vanishes, if the gap is positive. The stiffness $k$ should be large enough to forbid too large penetration, so as to satisfy some 'macroscopic' impenetrability.

In many circumstances, the adopted unilaterality description does not matter, provided that the penetration remains reasonably small. Most often, it is a matter of numerical convenience to adopt one or other law, the flexibility model, $R_{\mathrm{N}}=$ $-k g$, etc., being a regularized form of the Signorini condition, allowing us to use those special methods of non-smooth mechanics which are methods adapted from smooth mechanics. The above discussion, about unilaterality, holds for dry friction. Coulomb's law accounts for the main features of dry friction, i.e. sliding requires that tangential efforts exceed some threshold, the larger the normal pressure, the larger the threshold. Regularized forms of Coulomb's law may be relevant as well, and more sophisticated laws might be used, specially if tribological data are available. 
A cohesion phenomenon may be described as the property of two bodies in contact as long as separating forces do not exceed some threshold. There exist many physical mechanisms producing such an effect. At molecular scales, the Lennard-Jones law is commonly used, accounting for impenetrability, and describing tensile forces rapidly vanishing when the gap is increased (see figure $1 a$ and Rose et al. $(1981,1983)$ ). The relation between the normal reaction force and the gap is smooth but steep. Following the line of the above discussion, one may ignore those details describing the cohesive forces near the threshold at a fine scale and adopt a graph such as figure $1 b$, resulting from the graph in figure $1 a$ by a contraction of the gap scale. One might keep in mind the fact that the gap is positive, that the normal reaction force may not exceed some negative threshold, and that this force vanishes when the gap is strictly positive, data to be summarized on a 'limit graph'. In fact, a limit graph will not contain full data, and energy estimation is ambiguous. One is led to distinguish the history of the contacting bodies or left status before the contacting instant, and the right status,

if the left status is 'cohesive':

$g \geqslant 0, \quad R_{\mathrm{N}}+c \geqslant 0, \quad g\left(R_{\mathrm{N}}+c\right)=0$,

else:

$g \geqslant 0, \quad R_{\mathrm{N}} \geqslant 0, \quad g R_{\mathrm{N}}=0$,

the graphs of these relations being depicted in figure 2. These relations are written together with status change rules:

the right status is the same as the left status, except

(a) if the left status is 'cohesive', and if the solution $R_{\mathrm{N}}$ satisfies $R_{\mathrm{N}}+c<0$ or $g>0$,

then the right status is set to 'non-cohesive';

(b) if the left status is 'non-cohesive',

and if the solution satisfies $g \leqslant 0$,

then the right status is set to 'cohesive'.

This rule allows the contacting bodies to be glued again. Other status definitions and change rules may be introduced. For instance, the following rule allows bodies to be glued again only when some pressure is exerted:

(b) if the left status is 'non-cohesive', and if the solution satisfies $g \leqslant 0$ and $R_{\mathrm{N}}>0$,

then the right status is set to 'cohesive'.

Rule (b) may be omitted, which means that when separated, bodies cannot be glued again. More sophisticated cohesive models will be referred to in $\S 2$.

In the preceding, steep regular models were presented as opposed to strictly nonsmooth (non-differentiable) models, for instance, the flexibility model of unilateral constraints as opposed to the Signorini relation, the Lennard-Jones model as opposed to the Signorini-like condition monitored by the cohesive status. Though regularized 
models allow us to use the facilities of smooth methods, adapted for the circumstances, it happens that mere changes of variables allow us to apply a 'standard non-smooth contact dynamics (NSCD) method' to a wide range of models, so-called 'Signorini, Coulomb, derived' models, either steep regular models or strictly nonsmooth models, as will be developed in $\S 6$.

The NSCD method (Jean 1995, 1999; Jean \& Moreau 1992; Moreau 1994, 1999) does not use the facilities of regularized formulations, and rather in the line of convex analysis, uses a pair of relations: the Signorini condition (a complementary relation) and Coulomb's law. With this respect, the NSCD method has some relationship with Newton's generalized method (Alart \& Curnier 1988, 1991), mathematical programming methods (Chabrand et al. 1995; Klarbring 1990), and the bi-potential method (De Saxcé \& Feng 1991). Nevertheless, the NSCD solving procedure differs from those used in these methods. The main features of the standard NSCD method, are

(i) for each candidate to contact (a unique pair composed of a candidate body and of an antagonist body), the relative velocity and the reaction force are related through a unilateral Signorini-like relation and a frictional Coulomblike relation;

(ii) the relative velocity and the reaction force are also linearily related through a linearized form of the dynamical equation;

(iii) for each candidate to contact, assuming provisional values of the reaction forces at other candidates, a straightforward solution may be computed for the socalled 'Signorini, Coulomb, standard' problem defined in the above items;

(iv) the unknowns are updated from a candidate to the next candidate using the 'standard' solution; this updating procedure is similar to a nonlinear block Gauss-Seidel algorithm.

More details will be found in $\oint 5 a$. It is noteworthy that the NSCD method is fully implicit, while the usual regularized methods are fully explicit.

\section{Different approaches of cohesive laws}

Some particular cohesive laws are the so-called 'cohesive zone models'. These models relating reaction forces and displacement jumps across an interface, between two candidate bodies, or two candidate parts of the same body, are commonly used to describe the separation of some glued materials as well as the initiation and propagation of cracks. Fracture mechanics and cohesive zone models are two main approaches for analysing these crack initiations and propagations in materials.

Fracture mechanics is a classical tool of investigation, and particularly successful when applied to homogeneous elastic materials in quasi-static situations. It might fail in more complex circumstances, mainly when the question of initiation of cracks, space (branching) instability and time instability while growing arises. It might suffer also from the lack of ability to take into account complex physical phenomena such as frictional contact between the edges of a crack, wear, growth, coalescence, etc. Much effort has been put into these areas of research. But so far, either theoretically or numerically, describing a complete process of birth and growth of cracks is still an open problem. Nevertheless, fracture mechanics offers relevant energetic considerations, in particular, the energy release rate $G$. 
The cohesive zone models allow us to attack the question of birth and growth of cracks in complex situations, composite materials, highly heterogeneous microstructured materials, complex local loadings, three-dimensional cracks, instabilities, etc., while keeping in mind the physics at the local scale. Cohesive zone models allow us to mix several kinds of energy criterion, such as the critical energy release rate and the maximal stress criterion (mode II maximal shearing, etc.). Crack growth is described with those kinds of local criteria written with stresses and displacement jumps. Initiation and propagation should result from the knowledge of the loading path, without supplementary assumptions.

Cohesive zone models, initially introduced by Dugdale (1960), Barenblatt (1962) and Rice (1968), involved only the relations between normal stresses (traction or compression) and the opening (gap) at the edges of a crack. Later on, shearing was taken into account in the models of Ida (1972) on dynamical fracture in the Earth's crust and of Palmer \& Rice (1973) on the shearing of an inclined clay layer under gravity. Unilateral conditions were introduced later by Frémond (1982). The concept of cohesive domains was generalized in the late 1980s by Needleman (1987), who proposed phenomenological models of decohesion, independent of the loading path, suggested by the atomic scale researches of Rose et al. $(1981,1983)$ on bimetallic materials. These models, ready to become realistic crack models, took into account, as soon as 1990, the local irreversible behaviour, introducing surface damage (Needleman 1992; Tvergaard 1990). Considering the kinematics of the opening, in the normal direction as well as in the tangential direction (Tvergaard 1990) introduces a post-decohesion Coulomb frictional behaviour, and the bounding effect of residual stresses (Tvergaard 1991). After these pioneering works, recent analytical and numerical developments deal with quasi-static or dynamic propagation of cracks (Costanzo \& Walton 1997; Needleman \& Rosakis 1999; Xu \& Needleman 1994; Xu et al. 1998). A pair composed of an antagonist point and a candidate point to contact, in a discrete formulation, might be considered as well as a cohesive zone model. The cohesive zone model not only applies to cracks but also to bulk material. This idea has been applied in quite general situations: elasto-plasticity (Needleman 1990; Tvergaard \& Hutchinson 1992), dynamical propagation of cracks (Xu \& Needleman 1994), elasto-visco-plasticity (Siegmund \& Needleman 1997), and composite materials (Needleman \& Rosakis 1999; Siegmund et al. 1997; Xu et al. 1997). One may also quote the contributions of the French school on the micromechanical approach of cracks in composite materials (Cangémi et al. 1996; Chaboche et al. 1997; Frémond 1982; Michel \& Suquet 1994), and on damage in composite material plies (Allix et al. 1989, 1995).

\section{The basic equations}

Though emphasis is put on deformable bodies, the question of space discretization is not discussed in this paper. Finite-element methods are used. Discrete variables and equations governing the coordinates of the nodes will be written at once.

\section{(a) Notation}

For the sake of simplicity, it will be assumed that some nodes on a contacting meshed body are considered as the material points where the contact is to occur. 
To each candidate $\mathrm{P}$ is associated a unit vector $\boldsymbol{n}$ orthogonal to the boundary of the antagonist body, directed from the antagonist body to the candidate body. The material point $\mathrm{P}^{\prime}$ lying on the boundary of the antagonist body, and such that $\overrightarrow{\mathrm{P}^{\prime} \mathrm{P}}$ is collinear to $\boldsymbol{n}$ (i.e. the orthogonal projection of $\mathrm{P}$ on the antagonist boundary), is marked as the antagonist material point associated to P. An approximate boundary, or an approximate normal vector is often used, for convenience, and to ensure the uniqueness of the antagonist material point. In tricky circumstances, any reasonable algorithm to define an antagonist material point $\mathrm{P}^{\prime}$, 'proximal' to $\mathrm{P}$, will do. It will be referred to as a candidate for contact, as the unique pair of candidate antagonist bodies, or as the candidate antagonist material point, or merely as the candidate material point. An orthonormal basis $(\boldsymbol{t}, \boldsymbol{n}, \boldsymbol{s})$, referred to as the local frame, may be constructed with any two unit vectors $\boldsymbol{t}, \boldsymbol{s}$, orthogonal to each other and to $\boldsymbol{n}$. Normal components of vectors in the local frame will be denoted by the subscript ' $\mathrm{N}$ ', while the pair of tangential components will be denoted by the subscript ' $\mathrm{T}$ '. The relative velocity vector (in an extended sense) is defined as $\boldsymbol{U}=\boldsymbol{V}(\mathrm{P})-\boldsymbol{V}\left(\mathrm{P}^{\prime}\right)$, where $\boldsymbol{V}(\mathrm{P})$ is the velocity vector of the material point $\mathrm{P}$, and $\boldsymbol{V}\left(\mathrm{P}^{\prime}\right)$ is the velocity vector of the material point $\mathrm{P}^{\prime}$. The components of this vector in the local frame will be written as $U=\left(U_{\mathrm{T}}, U_{\mathrm{N}}\right)$. The reaction force (dual variable of $\boldsymbol{U}$ ), acting from the antagonist body to the candidate body, has components $R=\left(R_{\mathrm{T}}, R_{\mathrm{N}}\right)$ in the local frame. The gap is defined as $g=\overline{\mathrm{P}^{\prime} \mathrm{P}}$. It is strictly positive when bodies are not contacting, and strictly negative when they interpenetrate. The candidates to contact will be labelled with Greek superscripts, such as $\alpha$ and $\beta$. The number of candidates to contact is $\chi$.

\section{(b) Kinematic relations}

The variable $q \in \mathbb{R}^{N}$ is the (column) vector of nodes coordinates, $n$ being the number of nodes, $N=3 n$ being the number of degrees of freedom. If some degrees of freedom are imposed, penalization techniques are used. During motion, $q$ is a function of the time $t$. The time derivative of the mapping $t \rightarrow q(t)$ is denoted $\dot{q}$. There exists a linear mapping $G^{\alpha}(q)$ such that the relative velocity at the candidate $\alpha$ is given by the formula,

$$
U^{\alpha}=G^{\alpha}(q) \dot{q}
$$

For instance, if $\mathrm{P}$ is a node candidate to contact, $\mathrm{P}^{\prime}$ the antagonist particle lying on a mesh boundary line with end points the nodes $\mathrm{A}, \mathrm{B}$, the velocity of $\mathrm{P}^{\prime}$ is $\boldsymbol{V}\left(\mathrm{P}^{\prime}\right)=\left(\overline{\mathrm{AP}^{\prime}} / \overline{\mathrm{AB}}\right) \boldsymbol{V}(\mathrm{B})+\left(\overline{\mathrm{P}^{\prime} \mathrm{B}} / \overline{\mathrm{AB}}\right) \boldsymbol{V}(\mathrm{A})$, and $\boldsymbol{U}=\boldsymbol{V}(\mathrm{P})-\boldsymbol{V}\left(\mathrm{P}^{\prime}\right)$, which yields a formula as above. From duality considerations (conservation of the power), the node resulting force $r^{\alpha}$ corresponding to a local reaction force $R^{\alpha}$ satisfies

$$
\forall v, \quad G^{\alpha}(q) v \cdot R^{\alpha}=v \cdot G^{* \alpha}(q) R^{\alpha}=v \cdot r^{\alpha},
$$

where $G^{* \alpha}(q)=H^{\alpha}(q)$ is the linear transposed mapping of $G^{\alpha}(q)$. The usual notation in this paper is

$$
U^{\alpha}=H^{* \alpha}(q) \dot{q}, \quad r^{\alpha}=H^{\alpha}(q) R^{\alpha} .
$$

Another fundamental kinematic formula is that the derivative of the gap function $t \rightarrow g(t)$ is the normal component of the relative velocity,

$$
\dot{g}=U_{\mathrm{N}} .
$$


(c) The dynamical equation

The dynamical equation of the problem is written,

$$
M \ddot{q}=F_{\text {int }}(q, \dot{q})+P(t)+r,
$$

where $M$ is the mass matrix, $F_{\text {int }}(q, \dot{q})$ represents the node forces corresponding to internal efforts, $P(t)$ represents explicitly known excitation forces, and $r$ are the node forces corresponding to the reaction forces exerted at candidates to contact.

(d) The Signorini relation and the frictional Coulomb's law

The unilateral Signorini condition is written as

$$
g \geqslant 0, \quad R_{\mathrm{N}} \geqslant 0, \quad g R_{\mathrm{N}}=0 .
$$

Using 'standard variables', $\mathcal{U}_{\mathrm{N}}, \mathcal{R}_{\mathrm{N}}$, the complementary relation,

$$
\mathcal{U}_{\mathrm{N}} \geqslant 0, \quad \mathcal{R}_{\mathrm{N}} \geqslant 0, \quad \mathcal{U}_{\mathrm{N}} \mathcal{R}_{\mathrm{N}}=0
$$

or any equivalent form, will be referred to as the 'Signorini standard' relation,

$$
S_{\text {Sig }}\left(\mathcal{U}_{\mathrm{N}}, \mathcal{R}_{\mathrm{N}}\right)
$$

An equivalent form is

$$
\mathcal{R}_{\mathrm{N}}=\operatorname{proj}_{\mathbb{R}^{+}}\left(\mathcal{R}_{\mathrm{N}}-\rho \mathcal{U}_{\mathrm{N}}\right) \text {, where } \rho>0 \text { is arbitrary. }
$$

Coulomb's law is written as

$$
\left\|R_{\mathrm{T}}\right\| \leqslant \mu R_{\mathrm{N}}, \quad\left\|U_{\mathrm{T}}\right\| \neq 0 \Rightarrow R_{\mathrm{T}}=-\mu R_{\mathrm{N}} \frac{U_{\mathrm{T}}}{\left\|U_{\mathrm{T}}\right\|} .
$$

Using 'standard variables' $\mathcal{U}, \mathcal{R}$, the relation,

$$
\left\|\mathcal{R}_{\mathrm{T}}\right\| \leqslant \mu \mathcal{R}_{\mathrm{N}}, \quad\left\|\mathcal{U}_{\mathrm{T}}\right\| \neq 0 \Rightarrow \mathcal{R}_{\mathrm{T}}=-\mu \mathcal{R}_{\mathrm{N}} \frac{\mathcal{U}_{\mathrm{T}}}{\left\|\mathcal{U}_{\mathrm{T}}\right\|}
$$

or any equivalent form, will be referred to as the ' $\mu$-Coulomb standard' law,

$$
C_{\text {Coul } \mu \mathcal{R}_{\mathrm{N}}}\left(\mathcal{U}_{\mathrm{T}}, \mathcal{R}_{\mathrm{T}}\right)
$$

An equivalent form is

$$
\mathcal{R}_{\mathrm{T}}=\operatorname{proj}_{\mu \mathcal{R}_{\mathrm{N}} \mathbb{B}}\left(\mathcal{R}_{\mathrm{T}}-\rho \mathcal{U}_{\mathrm{T}}\right), \quad \text { where } \rho>0 \text { is arbitrary. }
$$

$\mathbb{B}$ is the unit disc with centre 0 in $\mathbb{R}^{2}$. Another equivalent form is expressed as a principle of minimal dissipation,

$$
\mathcal{R}_{\mathrm{T}} \in \mu \mathcal{R}_{\mathrm{N}} \mathbb{B}, \quad \forall \mathcal{S} \in \mu \mathcal{R}_{\mathrm{N}} \mathbb{B}, \quad \mathcal{U}_{\mathrm{T}} \cdot\left(\mathcal{S}-\mathcal{R}_{\mathrm{T}}\right) \geqslant 0
$$




\section{Time discretization}

(a) Numerical algorithm for the dynamical equation

When time discretization is performed, an elementary subinterval $\left.] t_{i}, t_{i+1}\right]$ of length $h$ is considered. The main idea developed in the process of time discretization is that discrete variables are not necessarily to be defined at some special time belonging to this interval, since the times where significant frictional effects occur are usually unknown, or costly to approximate, or even difficult to separate, when simultaneous contacts are occurring. Discrete frictional contact relations are thus defined 'over' the interval of time $\left.] t_{i}, t_{i+1}\right]$. Integrating both sides of the dynamical equation yields

$$
\left.\begin{array}{rl}
M\left(\dot{q}\left(t_{i+1}\right)-\dot{q}\left(t_{i}\right)\right) & =\int_{t_{i}}^{t_{i+1}} F(q, \dot{q}) \mathrm{d} s+\int_{] t_{i}, t_{i+1}\right]} P \mathrm{~d} t+\int_{] t_{i}, t_{i+1}\right]} r \mathrm{~d} \nu, \\
q\left(t_{i+1}\right) & =q\left(t_{i}\right)+\int_{t_{i}}^{t_{i+1}} \dot{q} \mathrm{~d} s .
\end{array}\right\}
$$

The mean value impulse denoted $r(i+1)$,

$$
r(i+1)=\frac{1}{h} \int_{] t_{i}, t_{i+1}\right]} r \mathrm{~d} \nu,
$$

emerges as a primary unknown. A typical numerical method is the $\theta$-method. Setting $\dot{q}(i), q(i), \dot{q}(i+1), q(i+1)$, approximations of $\dot{q}\left(t_{i}\right), q\left(t_{i}\right), \dot{q}\left(t_{i+1}\right), q\left(t_{i+1}\right)$, are

$$
\begin{aligned}
\int_{t_{i}}^{t_{i}+1} F(q, \dot{q}) \mathrm{d} s & \approx h \theta F(q(i+1), \dot{q}(i+1))+h(1-\theta) F(q(i), \dot{q}(i)), \\
q(i+1) & =q(i)+h \theta \dot{q}(i+1)+h(1-\theta) \dot{q}(i) .
\end{aligned}
$$

Since, in this paper, emphasis is put on cohesive frictional laws, it will be supposed that materials are elastic within the assumption of small perturbations. Thus the technical details of nesting some Newton-Raphson loop will be avoided, and introduced only if the material behaviour law is nonlinear (see Jean 1999). Assuming an elastic linear behaviour,

$$
F(q, \dot{q})=-V \dot{q}-K q,
$$

where $V$ is the damping matrix and $K$ is the stiffness matrix, equation (4.1) is written as

$$
\begin{aligned}
M(\dot{q}(i+1)-\dot{q}(i))=h \theta( & \left.-V \dot{q}(i+1)-K q(i+1)+P\left(t_{i+1}\right)\right) \\
& +h(1-\theta)\left(-V \dot{q}(i)-K q(i)+P\left(t_{i}\right)\right)+h r(i+1),
\end{aligned}
$$

and the $\theta$-algorithm is

$$
\begin{aligned}
\dot{q}(i+1)-\dot{q}(i) & =w(-h V \dot{q}(i)-h K(q(i)+\theta h \dot{q}(i))+h P(i+1)+h r(i+1)), \\
q(i+1) & =q(i)+h \theta \dot{q}(i+1)+h(1-\theta) \dot{q}(i), \\
\text { where } & \\
w & =\left(M+h \theta V+h^{2} \theta^{2} K\right)^{-1} \\
P(i+1) & =\theta P\left(t_{i+1}\right)+(1-\theta) P\left(t_{i}\right) .
\end{aligned}
$$


It is assumed that the matrix $M+h \theta V+h^{2} \theta^{2} K$ is one-to-one, which is satisfied if $h$ is small enough, the mass matrix $M$ being positive definite. When non-smooth mechanics effects, shocks or changes in frictional contact status occur, generating velocities discontinuities, first-order schemes are sufficient. Higher-order approximations schemes are irrelevant and even troublesome, since they require appropriate regularity properties which are not met. The above formula, a linearized form of the discrete dynamical equation, appears as an affine relation between two primary unknowns, the velocity $\dot{q}(i+1)$ and the mean value impulse $r(i+1)$,

$$
\dot{q}(i+1)=v_{\text {free }}(i)+w h r(i+1) .
$$

Many numerical algorithms may be written under such a form. The NSCD method is based on the use of such an affine relation, whatever the choice of the approximation process for the dynamical equation. In the following, for the sake of simplicity, the choice $\theta=1$ is made, i.e. the choice corresponding to the implicit Euler method.

\section{(b) Discrete forms of kinematic relations}

The adopted discrete forms of (3.1) are

$$
U^{\alpha}(i+1)=H^{* \alpha}(\hat{q}) \dot{q}(i+1), \quad r^{\alpha}(i+1)=H^{\alpha}(\hat{q}) R^{\alpha}(i+1) .
$$

The variable $\hat{q}$ denotes the configuration used to define the local frames. In a fully implicit method, the choice $\hat{q}=q(i+1)$ should be made. This complication is unnecessary as far as the lengths $h U^{\alpha}(i+1)$ remain sufficiently small with respect to curvature radius of contacting bodies. Actually, the choice $\hat{q}=q(i)$ is easy and fair enough. In the following the variable $\hat{q}$ is omitted:

$$
U^{\alpha}(i+1)=H^{* \alpha} \dot{q}(i+1), \quad r^{\alpha}(i+1)=H^{\alpha} R^{\alpha}(i+1) .
$$

Denoting $\dot{g}^{\alpha}(i+1)$ an approximation of $\dot{g}^{\alpha}\left(t_{i+1}\right)$, the formula (3.2) can be written,

$$
\dot{g}^{\alpha}(i+1)=U_{\mathrm{N}}^{\alpha}(i+1) .
$$

This formula suggests the predictive formula,

$$
g^{\alpha}(i+1)=g^{\alpha}(i)+h U_{\mathrm{N}}^{\alpha}(i+1) .
$$

The choice $\theta=1$ has been made for the sake of simplicity. Incidentally, it is worth drawing attention to the general case $0.5 \leqslant \theta<1$. Expressing unilateral conditions with predictive gaps at times $t_{i}+(1-\theta) h, t_{i+1}+(1-\theta) h$, i.e. $\theta$-midpoints, instead of $t_{i+1}$, the end of the time-step, is recommended, with a predictive formula such as

$$
\begin{aligned}
g^{\alpha}(1-\theta+i) & =g^{\alpha}(i)+(1-\theta) h U_{\mathrm{N}}^{\alpha}(i), \\
g^{\alpha}(1-\theta+i+1) & =g^{\alpha}(i+1)+(1-\theta) h U_{\mathrm{N}}^{\alpha}(i+1) .
\end{aligned}
$$

Indeed, unilateral conditions imply a contradictory rule to be satisfied both by the relative velocity and the gap at the end of the time-step (except in the case $\theta=1$ ). Oscillatory artefacts might be generated. For more details see a discussion about consistency in Jean (1999) and Vola et al. (1998). 
(c) Discrete forms of the Signorini relation and Coulomb's law

The Signorini relation graph is infinitely steep, and as a consequence the gap $g$ cannot be written as a single-valued mapping of $R_{\mathrm{N}}$, nor $R_{\mathrm{N}}$ as a single-valued mapping of $g$. This leads us to adopt fully implicit schemes and to choose both the values of $g^{\alpha}(i+1)$ at the end of the time-step, and $R_{\mathrm{N}}^{\alpha}(i+1)$ the mean value impulse, as unknowns in the frictional contact laws. For instance the discrete form of the Signorini relation (3.4) is

$$
S_{\mathrm{Sig}}\left(g^{\alpha}(i+1), R_{\mathrm{N}}^{\alpha}(i+1)\right) .
$$

For similar reasons, the discrete form of Coulomb's law (3.7) is

$$
C_{\text {Coul } \mu^{\alpha} R_{\mathrm{N}}^{\alpha}(i+1)}\left(U_{\mathrm{T}}^{\alpha}(i+1), R_{\mathrm{T}}^{\alpha}(i+1)\right) .
$$

\section{The NSCD approach}

(a) The 'Signorini, $\mu$-Coulomb, standard' problem

As an example consider the 'ordinary' dynamical frictional contact problem, governed by the dynamical equation (4.4), the kinematic relations (4.5), the Signorini, Coulomb's relation, (4.8), (4.9).

Using the kinematic relations allows us to write a linearized form of the dynamical equation (4.4) in terms of the local variables, the relative velocity $U^{\alpha}(i+1)$ and the reaction force $R^{\alpha}(i+1)$, at the candidate to contact $\alpha$,

$$
\begin{aligned}
U^{\alpha}(i+1) & =U_{\text {free }}^{\alpha}+\sum_{\beta} W^{\alpha \beta} h R^{\beta}(i+1), \\
W^{\alpha \beta} & =H^{* \alpha} w H^{\beta} \\
U_{\text {free }}^{\alpha} & =H^{* \alpha} v_{\text {free }} .
\end{aligned}
$$

Assuming some provisional values for $R^{\beta}(i+1)$, the above equation becomes

$$
U^{\alpha}(i+1)=U_{\text {loc free }}^{\alpha}+W^{\alpha \alpha} h R^{\alpha}(i+1),
$$

where

$$
U_{\text {loc free }}^{\alpha}=U_{\text {free }}^{\alpha}+\sum_{\beta \neq \alpha} W^{\alpha \beta} h R^{\beta}(i+1) .
$$

This equation has to be written together with a discrete form of the Signorini relation, and a discrete form of Coulomb's law, together with the kinematic relation (4.7). Setting

$$
\begin{aligned}
\bar{U}_{\text {loc free } \mathrm{T}}^{\alpha}=U_{\text {loc free T }}^{\alpha}, \quad \bar{U}_{\text {loc free } \mathrm{N}}^{\alpha} & =U_{\text {loc free } \mathrm{N}}^{\alpha}+\frac{1}{h} g^{\alpha}(i), \\
\bar{W}^{\alpha \alpha}=W^{\alpha \alpha}, \quad \mu & =\mu^{\alpha},
\end{aligned}
$$

it is found that the unknowns,

$$
\begin{gathered}
\bar{U}_{\mathrm{T}}=U_{\mathrm{T}}^{\alpha}(i+1), \quad \bar{U}_{\mathrm{N}}=\frac{1}{h} g^{\alpha}(i+1), \\
\bar{R}=R^{\alpha}(i+1),
\end{gathered}
$$


are solutions of the following 'Signorini, $\mu$-Coulomb, standard' problem:

$$
\left.\begin{array}{c}
\text { find } \mathcal{U}, \mathcal{R}, \text { such that } \\
\mathcal{U}=\mathcal{U}_{\text {loc free }}+\mathcal{W} h \mathcal{R}, \\
S_{\mathrm{Sig}}\left(\mathcal{U}_{\mathrm{N}}, \mathcal{R}_{\mathrm{N}}\right), \\
C_{\text {Coul } \mu \mathcal{R}_{\mathrm{N}}}\left(\mathcal{U}_{\mathrm{T}}, \mathcal{R}_{\mathrm{T}}\right),
\end{array}\right\}
$$

where $\mathcal{U}, \mathcal{R}, \mathcal{U}_{\text {loc free }}=\bar{U}_{\text {loc free }}^{\alpha}$ (some data), belong to $\mathbb{R}^{3}\left(\mathbb{R}^{2}\right.$ in the two-dimensional case), $\mathcal{W}=\bar{W}^{\alpha \alpha}$ (an element of data), is a $3 \times 3$ matrix $(2 \times 2$ in the two-dimensional case). The solution of this elementary problem is found by investigating the intersection of piecewise affine mappings, since the Signorini relation and Coulomb's law appear in such a form (see (3.6), (3.9)). It happens that in the two-dimensional case, the elementary standard solution can be explicitly written (see $\S 9$ and Jean (1999)). In the three-dimensional case, a few iterations of a generalized Newton-Raphson method allow super convergence toward the elementary standard solution (Alart \& Curnier 1988). The NSCD algorithm is as follows.

\section{(b) Solving the frictional contact problem}

The unknowns $R^{\alpha}(i+1)$, the mean value impulses at step $i, i+1$, are sought as the limit of sequences $R^{\alpha}(p), p=1, \ldots, p_{\max }$, where $p_{\max }$ is maximum number of iterations:

(1) at iteration $p+1$, seek an approximate solution at candidate $\alpha$; provisional values at other candidates are adopted as follows: if $\beta>\alpha$, these are the values computed at iteration $p$, and if $\beta<\alpha$, these are the values just computed at iteration $p+1$,

$$
R^{\beta}(p+1), \beta=1, \ldots, \alpha-1, \quad R^{\beta}(p), \beta=\alpha+1, \ldots, \chi
$$

the free velocity (i.e. the relative velocity at candidate $\alpha$ equipped with a vanishing reaction), while provisional reactions are exerted on neighbouring candidates, is computed,

$$
U_{\text {loc free }}^{\alpha}=U_{\text {free }}^{\alpha}+\sum_{\beta<\alpha} W^{\alpha \beta} h R^{\beta}(p+1)+\sum_{\beta>\alpha} W^{\alpha \beta} h R^{\beta}(p),
$$

(a) compute or call,

$$
\bar{U}_{\text {loc free T }}^{\alpha}=U_{\text {loc free } \mathrm{T}}^{\alpha}, \quad \bar{U}_{\text {loc free } \mathrm{N}}^{\alpha}=U_{\text {loc free } \mathrm{N}}^{\alpha}+\frac{1}{h} g^{\alpha}(i), \quad \bar{W}=W^{\alpha \alpha} ;
$$

(b) find a solution $\bar{U}, \bar{R}$, of the 'Signorini, $\mu$-Coulomb, standard' problem (5.5), with the data $\mathcal{U}_{\text {loc free }}=\bar{U}_{\text {loc free }}^{\alpha}, \mathcal{W}=\bar{W}^{\alpha \alpha}, \mu=\mu^{\alpha}$;

(c) set $R^{\alpha}(p+1)=\bar{R}$;

(2) reactions are updated and the next candidate is called;

(3) the list of candidates is read ( $p$ is increased) until some quality criterion is satisfied or $p=p_{\max }$.

This method is similar to a nonlinear block Gauss-Seidel method. 


\section{Derived 'Signorini, $\mu$-Coulomb, standard' laws}

In this section the concept of 'Signorini, $\mu$-Coulomb, derived' law, is introduced: some variables, images of the local basic variables, gap, relative velocity, reaction force, by some affine mappings, satisfy the standard Signorini relation and Coulomb's law. Such changes of variables are defined at the beginning of the time-step by some status, a data storage that is supposed to summarize the significant history of contact.

\section{(a) Derived law}

A Signorini, $\mu$-Coulomb, derived law is defined by

1. a status variable, $S_{\text {status }}^{\alpha}(i)$, entirely defined by the relative velocity, the gap, the mean value of the impulse, $U^{\alpha}(i), g^{\alpha}(i), R^{\alpha}(i)$, and possibly status at previous time-steps $j<i$;

2. auxiliary unknowns, $\bar{U}, \bar{R}$, affine functions of $U^{\alpha}(i+1), g^{\alpha}(i+1), R^{\alpha}(i+1)$; the coefficients of these functions depend on the status $S_{\text {status }}^{\alpha}(i)$ at step $i$, and $U^{\alpha}(i), g^{\alpha}(i), R^{\alpha}(i)$;

3. according to the status, either $R^{\alpha}(i+1)=0$, or the auxiliary variables $\bar{U}, \bar{R}$, satisfy 'Signorini, $\mu$-Coulomb, standard' law, (3.5), (3.8).

\section{(b) Change of variables}

The index $\alpha$ is momentarily omitted. It has been seen that the relative velocity and the reaction force are related through the linearized dynamical equation (5.4),

$$
U(i+1)=U_{\mathrm{loc}} \text { free }+W h R(i+1) .
$$

The gap is given by the predictive formula (4.7),

$$
g(i+1)=g(i)+h U_{\mathrm{N}}(i+1) .
$$

The auxiliary unknowns $\bar{U}, \bar{R}$ are introduced as affine mappings of $U(i+1), g(i+1)$, $R(i+1)$, written as

$$
\left(\begin{array}{c}
\bar{U} \\
h \bar{R}
\end{array}\right)=\left(\begin{array}{cc}
I_{U U} & I_{U R} \\
I_{R U} & I_{R R}
\end{array}\right)\left(\begin{array}{c}
U(i+1) \\
h R(i+1)
\end{array}\right)+\left(\begin{array}{c}
\bar{U}_{0} \\
h \bar{R}_{0}
\end{array}\right) .
$$

It is supposed that the matrix,

$$
\left(\begin{array}{ll}
I_{U U} & I_{U R} \\
I_{R U} & I_{R R}
\end{array}\right)
$$

is one-to-one. An equivalent form of the previous relation is then

$$
\left(\begin{array}{c}
U(i+1) \\
h R(i+1)
\end{array}\right)=\left(\begin{array}{ll}
J_{U U} & J_{U R} \\
J_{R U} & J_{R R}
\end{array}\right)\left(\begin{array}{c}
\bar{U} \\
h \bar{R}
\end{array}\right)+\left(\begin{array}{c}
U_{0} \\
h R_{0}
\end{array}\right) .
$$

Thus

$$
\left(J_{U U}-W J_{R U}\right) U^{-}=\left(W J_{R R}-J_{U R}\right) h R^{-}+W h R_{0}-U_{0}+U_{\text {loc free }}
$$


It is supposed that the matrix $J_{U U}-W J_{R U}$ is one-to-one. The previous relation becomes

$$
\bar{U}=\bar{U}_{\text {loc free }}+\bar{W} h \bar{R}
$$

where

$$
\begin{aligned}
\bar{U}_{\text {loc free }} & =U_{\text {change }}+C_{\text {change }} U_{\text {loc free }}, \\
\bar{W} & =C_{\text {change }}\left(W J_{R R}-J_{U R}\right), \\
U_{\text {change }} & =C_{\text {change }}\left(W h R_{0}-U_{0}\right), \\
C_{\text {change }} & =\left(J_{U U}-W J_{R U}\right)^{-1} .
\end{aligned}
$$

The class of change of variables must be restricted so as to satisfy the objectivity principle as well as thermodynamic principles.

It should be noticed that the data $J_{U U}, J_{U R}, J_{R U}, J_{R R}, U_{0}, h R_{0}$, the matrices $\bar{W}, C_{\text {change }}$, the vector $U_{\text {change }}$, constructed with physical constants, depend only on $S_{\text {status }}^{\alpha}(i)$ and $U^{\alpha}(i), g^{\alpha}(i), R^{\alpha}(i)$. These data are to be prepared at the beginning of the time-step, before undergoing iterations. Equation (6.5) is the corresponding form of the linearized dynamical equation (6.1) through changes of variables (6.3), $(6.4)$.

\section{(c) Solving the frictional contact problem with a derived law}

The algorithm $\S 6 b$ is easily generalized.

Prepare the data

$$
\begin{gathered}
J_{U U}^{\alpha}, \quad J_{U R}^{\alpha}, \quad J_{R U}^{\alpha}, \quad J_{R R}^{\alpha}, U_{0}^{\alpha}, \quad h R_{0}^{\alpha}, \quad C_{\text {change }}^{\alpha}, U_{\text {change }}^{\alpha}, \\
\bar{W}^{\alpha \alpha}=C_{\text {change }}^{\alpha}\left(W^{\alpha \alpha} J_{R R}^{\alpha}-J_{U R}^{\alpha}\right),
\end{gathered}
$$

for all $\alpha$ and status $S_{\text {status }}^{\alpha}(i)$. The unknowns $R^{\alpha}(i+1)$ are sought as limits of the sequences $R^{\alpha}(p), p=1, \ldots, p_{\max }$ :

(1) at iteration $p+1$, seek an approximate solution at candidate $\alpha$; provisional values at other candidates are adopted as follows: if $\beta>\alpha$, these are the values computed at iteration $p$, and if $\beta<\alpha$, these are the values just computed at iteration $p+1$,

$$
R^{\beta}(p+1), \beta=1, \ldots, \alpha-1, \quad R^{\beta}(p), \beta=\alpha+1, \ldots, \chi ;
$$

compute the free velocity,

$$
U_{\text {loc free }}^{\alpha}=U_{\text {free }}^{\alpha}+\sum_{\beta<\alpha} W^{\alpha \beta} h R^{\beta}(p+1)+\sum_{\beta>\alpha} W^{\alpha \beta} h R^{\beta}(p) ;
$$

(a) according to $S_{\text {status }}^{\alpha}(i)$, compute $\bar{U}_{\text {loc free }}^{\alpha}=U_{\text {change }}^{\alpha}+C_{\text {change }}^{\alpha} U_{\text {loc free }}^{\alpha}$;

(b) find a solution $\bar{U}, \bar{R}$, of the 'Signorini, $\mu$-Coulomb, standard' problem (5.5), with the data $\mathcal{U}_{\text {loc free }}=\bar{U}_{\text {loc free }}^{\alpha}, \mathcal{W}=\bar{W}^{\alpha \alpha}, \mu=\mu^{\alpha}$

(c) make the reverse change of variables (6.4) to obtain $R^{\alpha}(p+1)$ from $\bar{R}$,

$$
\left(\begin{array}{c}
U^{\alpha}(i+1) \\
h R^{\alpha}(i+1)
\end{array}\right)=\left(\begin{array}{cc}
J_{U U}^{\alpha} & J_{U R}^{\alpha} \\
J_{R U}^{\alpha} & J_{R R}^{\alpha}
\end{array}\right)\left(\begin{array}{c}
\bar{U} \\
h \bar{R}
\end{array}\right)+\left(\begin{array}{c}
U_{0}^{\alpha} \\
h R_{0}^{\alpha}
\end{array}\right)
$$


(2) reactions are updated and the next candidate is called;

(3) the list of candidates is read ( $p$ is increased) until some quality criterion is satisfied or $p=p_{\max }$.

The 'ordinary' frictional contact problem, presented as an introductory example in $\S 5$, using (4.8), (4.9), may be considered as a special case, where a default status is assumed.

\section{(d) Non-frictional cohesive example}

As a second example, the NSCD method is applied to the non-frictional cohesive law already presented in $\S 1$.

Prepare the data:

(1) if $S_{\text {status }}^{\alpha}(i)$ is 'non-cohesive', the following change of variables is adopted, i.e. the 'ordinary' unilateral, frictional model (4.8), (4.9) is used,

$$
\begin{gathered}
\bar{U}_{\mathrm{T}}=U_{\mathrm{T}}^{\alpha}(i+1), \quad \bar{U}_{\mathrm{N}}=\frac{1}{h} g^{\alpha}(i+1), \\
\bar{R}=R^{\alpha}(i+1), \\
\mu^{\alpha}=0, \quad \mu^{\alpha} \text { friction coefficient. }
\end{gathered}
$$

It follows that

$$
\bar{U}_{\text {loc free } \mathrm{T}}^{\alpha}=U_{\text {loc free } \mathrm{T}}^{\alpha}, \quad \bar{U}_{\text {loc free } \mathrm{N}}^{\alpha}=U_{\text {loc free } \mathrm{N}}^{\alpha}+\frac{1}{h} g^{\alpha}(i), \quad \bar{W}^{\alpha \alpha}=W^{\alpha \alpha} ;
$$

(2) if $S_{\text {status }}^{\alpha}(i)$ is 'cohesive', the following change of variables is adopted,

$$
\begin{array}{rlrl}
\bar{U}_{\mathrm{T}} & =U_{\mathrm{T}}^{\alpha}(i+1), & \bar{U}_{\mathrm{N}}=\frac{1}{h} g^{\alpha}(i+1), \\
\bar{R}_{\mathrm{T}} & =R_{\mathrm{T}}^{\alpha}(i+1), & \bar{R}_{\mathrm{N}}=R_{\mathrm{N}}^{\alpha}(i+1)+c^{\alpha}, \\
c^{\alpha}>0, & c^{\alpha} \text { cohesion constant }, \\
\mu^{\alpha}=0, & \mu^{\alpha} \text { friction coefficient. }
\end{array}
$$

Setting

$$
W^{\alpha \alpha}=\left(\begin{array}{ll}
W_{\mathrm{TT}}^{\alpha \alpha} & W_{\mathrm{TN}}^{\alpha \alpha} \\
W_{\mathrm{NT}}^{\alpha \alpha} & W_{\mathrm{NN}}^{\alpha \alpha}
\end{array}\right)
$$

it follows that

$$
\begin{gathered}
\bar{U}_{\text {loc free } \mathrm{T}}^{\alpha}=U_{\text {loc free } \mathrm{T}}^{\alpha}-W_{\mathrm{TN}}^{\alpha \alpha} c^{\alpha}, \quad \bar{U}_{\text {loc free } \mathrm{N}}^{\alpha}=U_{\mathrm{loc} \text { free } \mathrm{N}}^{\alpha}+\frac{1}{h} g^{\alpha}(i)-W_{\mathrm{NN}}^{\alpha \alpha} c^{\alpha}, \\
\bar{W}^{\alpha \alpha}=W^{\alpha \alpha} .
\end{gathered}
$$

Some status change rules have to be chosen. The following rules are adopted:

$S_{\text {status }}^{\alpha}(i+1)$ is the same as $S_{\text {status }}^{\alpha}(i)$,

except

if $S_{\text {status }}^{\alpha}(i)$ is 'cohesive',

and if the solution $R_{\mathrm{N}}^{\alpha}(i+1)$ satisfies $R_{\mathrm{N}}^{\alpha}(i+1)+c^{\alpha}<0$, or $g^{\alpha}(i+1)>0$, then $S_{\text {status }}^{\alpha}(i+1)$ is set to 'non-cohesive'. 
When candidates to contact have been separated, the candidates are not allowed to be glued again. This is the rule in the next applications, $\S \S 7$ and 8. Other rules may be adopted.

The case of Mohr-Coulomb law is a mere generalization of the above case. One has only to set $\mu$ at the value of the friction coefficient $\mu^{\alpha}$ in the non-cohesive case, and to set $\mu=\operatorname{tg}\left(\varphi^{\alpha}\right)$ in the cohesive case, where $\varphi^{\alpha}$ is the internal friction angle. The change of status rule is

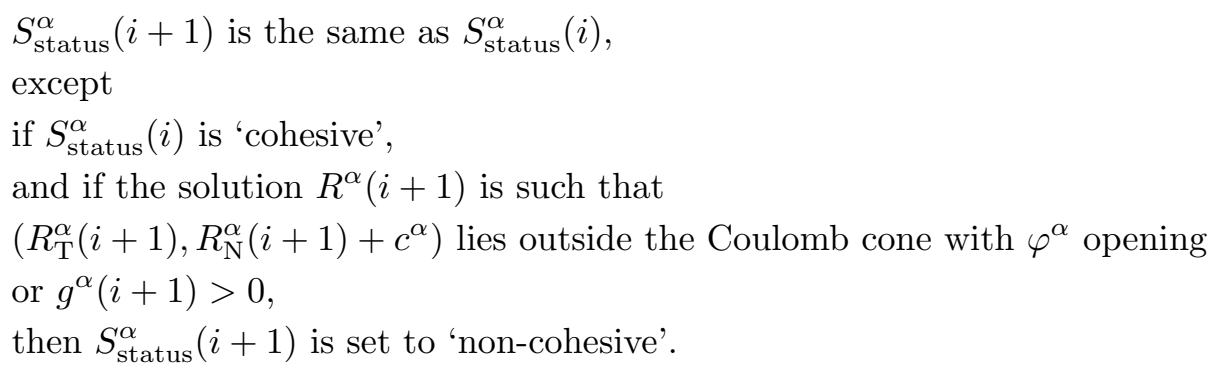

One sees that many laws may be constructed, playing with affine changes of variables and status change rules. Definitions of status may also be refined. This method applies as well to finite-elements models of contacting bodies as to collections of rigid bodies as granular materials. More details may be found in Cambou \& Jean (2001). As a matter of fact, if the use of (3.4), (3.7) is relevant when one of the contacting bodies is deformable, shock laws are to be used when both contacting bodies are rigid.

\section{(e) The Frémond-Cangemi-Raous cohesive model}

The previous model, the Mohr-Coulomb model, is 'very' non-smooth. Nevertheless, it is extensively used in soil mechanics, with some refinement, for instance, the Cam clay law or the Cambou-Jafari-Sidoroff model. The concept of damage is often related to some decohesive process. Refining the time-scale, introducing damage, is a way of describing more precisely the evolution of interface stresses when decohesion is occurring, i.e. going from undamaged to completely damaged interfaces. Such models are smoother than the mere Mohr-Coulomb model. Furthermore, it is possible to derive such models from thermodynamic considerations. The energy balance between the different contributions is thus easy to identify. In comparison, global energy estimations are possible with 'Signorini, $\mu$-Coulomb, derived' laws, as it is possible for shock laws, but the way the energy is dissipated is deliberately ignored.

The following Frémond-Cangemi-Raous (FCR) model, based on a thermodynamic approach (see Cangémi et al. 1996; Raous et al. 1997, 1999) belongs to the class of smoothed models in the above sense, i.e. a surface damage variable is introduced, together with a damage law governing the evolution of the damage variable. Strong velocity variations or discontinuities are expected during the decohesive process and dynamics of contacting particles should be taken into consideration. It is possible to admit the FCR model in the class of 'Signorini, $\mu$-Coulomb, derived' laws, allowing us to use the implicit NSCD method straightforwardly. The FCR model is hereafter presented in this context. At first an intermediate model is considered. The index $\alpha$ is omitted for simplicity.

Prepare the data: 


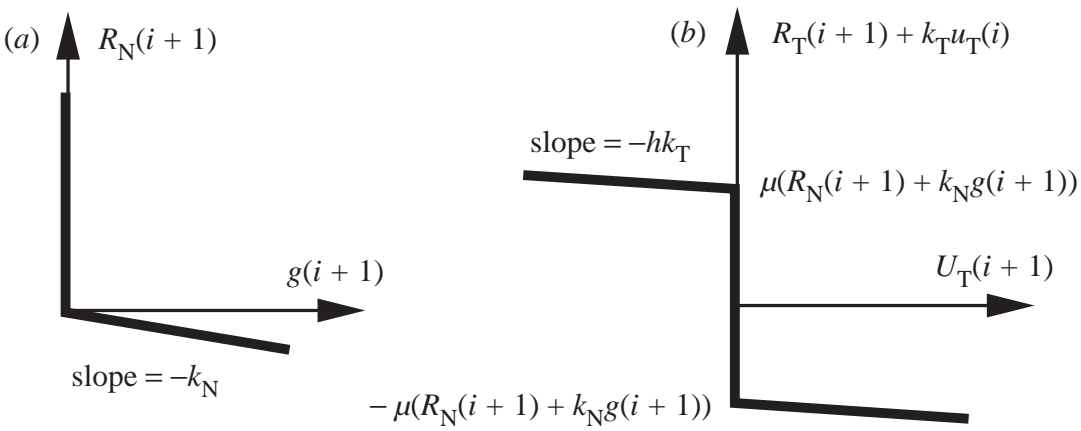

Figure 3. (a) Unilateral condition; (b) friction law.

(1) if $S_{\text {status }}(i)$ is 'non-cohesive', the following change of variables is adopted, i.e. the 'ordinary' unilateral, frictional model (4.8), (4.9) is used,

$$
\begin{gathered}
\bar{U}_{\mathrm{T}}=U_{\mathrm{T}}(i+1), \quad \bar{U}_{\mathrm{N}}=\frac{1}{h} g(i+1), \\
\bar{R}=R(i+1),
\end{gathered}
$$

$\mu$ is the non-cohesive status friction coefficient,

(2) if $S_{\text {status }}(i)$ is 'cohesive', the following change of variables is adopted,

$$
\begin{gathered}
\bar{U}_{\mathrm{T}}=U_{\mathrm{T}}(i+1), \quad \bar{U}_{\mathrm{N}}=\frac{1}{h} g(i+1), \\
\bar{R}_{\mathrm{T}}=R_{\mathrm{T}}(i+1)-k_{\mathrm{T}} u_{\mathrm{T}}(i+1), \\
\bar{R}_{\mathrm{N}}=R_{\mathrm{N}}(i+1)-k_{\mathrm{N}} g(i+1),
\end{gathered}
$$

$\mu$ is the cohesive status friction coefficient.

The symbols $k_{\mathrm{T}}, k_{\mathrm{N}}$ are stiffness constants (depending on $\alpha$ ). The variable $u_{\mathrm{T}}$ is the 'tangential displacement', the definition of which is

$$
u_{\mathrm{T}}(t)=\int_{] t_{0}, t\right]} U_{\mathrm{T}}(s) \mathrm{d} s,
$$

where $t_{0}$ is the instant where the contact is set. Actually, the definition and the numerical estimation of the tangential displacement give rise to serious difficulties. Within the small perturbations assumption, with finite sliding between small curvature bodies, the following approximate formula may be adopted,

$$
u_{\mathrm{T}}(i+i)=u_{\mathrm{T}}(i)+h U_{\mathrm{T}}(i+1),
$$

so that the above formula quoted $(\diamond)$ may be written as

$$
\bar{R}_{\mathrm{T}}=R_{\mathrm{T}}(i+1)-k_{\mathrm{T}} u_{\mathrm{T}}(i)-h k_{\mathrm{T}} U_{\mathrm{T}}(i+1) .
$$

If the variables $\bar{U}, \bar{R}$, satisfy the 'Signorini, $\mu$-Coulomb, standard' law, the variables, $R_{\mathrm{N}}(i+1),(1 / h) g(i+1)$ lie on the graph in figure $3 a$, and the variables $R_{\mathrm{T}}(i+1)$, $U_{\mathrm{T}}(i+1)$ lie on the graph in figure $3 b$. It means that, when the status is cohesive, some spring with stiffness $k_{\mathrm{N}}$ is acting from the actual antagonist point to the actual 
candidate point in the normal direction to prevent separation, while some spring with stiffness $k_{\mathrm{T}}$ is attached from the initial antagonist point (at $t_{0}$ ) to the actual candidate frictional point in the tangential direction. The FCR model is more sophisticated in the sense that the stiffnesses $k_{\mathrm{N}}, k_{\mathrm{T}}$ deteriorate according to some damage variable $\beta$ governed by a differential equation, with discrete form,

$$
k_{\mathrm{T}}=\beta(i+1)^{2} C_{\mathrm{T}}, \quad k_{\mathrm{N}}=\beta(i+1)^{2} C_{\mathrm{N}} .
$$

$\beta(i+1)$ is an approximate solution of the differential equation,

$$
\begin{aligned}
& \dot{\beta}=-\left(\frac{1}{b}\left[E G^{\prime}(\beta)-\beta\left(C_{\mathrm{N}} g^{2}+C_{\mathrm{T}} u_{\mathrm{T}}^{2}\right)\right]^{-}\right)^{1 / p}, \quad \text { if } \beta \in[0,1[, \\
& \dot{\beta} \leqslant-\left(\frac{1}{b}\left[E G^{\prime}(\beta)-\beta\left(C_{\mathrm{N}} g^{2}+C_{\mathrm{T}} u_{\mathrm{T}}^{2}\right)\right]^{-}\right)^{1 / p}, \quad \text { if } \beta=1 .
\end{aligned}
$$

The notation $[x]^{-}$stands for the negative part of $x,[x]^{-}=\max (0,-x)$, and $u_{\mathrm{T}}^{2}(i)$ stands for $u_{\mathrm{T}} \cdot u_{\mathrm{T}}$. The symbols $E, b, C_{\mathrm{T}}, C_{\mathrm{N}}$ are physical constants (depending on $\alpha$ ). The variable $\beta \in[0,1]$ is the damage variable. If $\beta=0$, the interface in the vicinity of the candidate to contact is undamaged, and is fully damaged if $\beta=1$. For the sake of simplicity, assume $G^{\prime}(\beta(i))=1$. The symbol $E$ is some reference energy. One sees from the above formula that as soon as the spring's elastic energy, $\frac{1}{2}\left(C_{\mathrm{N}} g^{2}+C_{\mathrm{T}} u_{\mathrm{T}}^{2}\right)$, reaches the value $\frac{1}{2} E$, the damage variable begins to decrease and keeps on decreasing as long as $\beta^{2} \frac{1}{2}\left(C_{\mathrm{N}} g^{2}+C_{\mathrm{T}} u_{\mathrm{T}}^{2}\right) \geqslant E \beta$. The status change rule is: if $\beta(i+1) \leqslant 0$, then $S_{\text {status }}(i+1)$ is set to 'non-cohesive'. In fact the 'cohesive' behaviour law degenerates as the 'non-cohesive' law as soon as the damage is achieved, $\beta=0$. The approximate value $\beta(i+1)$ may be found using an explicit scheme. Better results are obtained using an implicit $\theta$-scheme, which is possible without extra cost when outer Newton-Raphson loops are to be used.

The following example shows the possibilities of the FCR model operated by the NSCD method.

\section{An example of fibre-reinforced material}

Figure 4 illustrates a typical behaviour of a ceramic matrix composite material $(\mathrm{SiC} / \mathrm{SiC})$, reinforced with long fibres. Half a representative cell, including matrix and fibre of diameter of $c a .15 \mu \mathrm{m}$, is submitted to periodic limit conditions allowing us to account for the effects of a crack within the matrix, lying in a plane orthogonal to the fibres bundle. This configuration, together with the FCR model, allows us to analyse three main phenomena physically observed during the crack process: the crack propagates around the fibres and along the fibre-matrix interface, the crack is bridged by fibres; the crack propagates more slowly or even stops, being trapped by the fibres bundle; the crack breaks through matrix and fibres. These intrinsically three-dimensional phenomena depend on the respective properties of the matrix-fibre, matrix-matrix and fibre-fibre interfaces, some of them happening to be optimal from the point of view of the ability of the composite material to overcome crack propagation.

In figure 4, the cell is submitted to given tensile displacements imposed to the upper section in the direction of the fibres. Different physical constants are used to model 


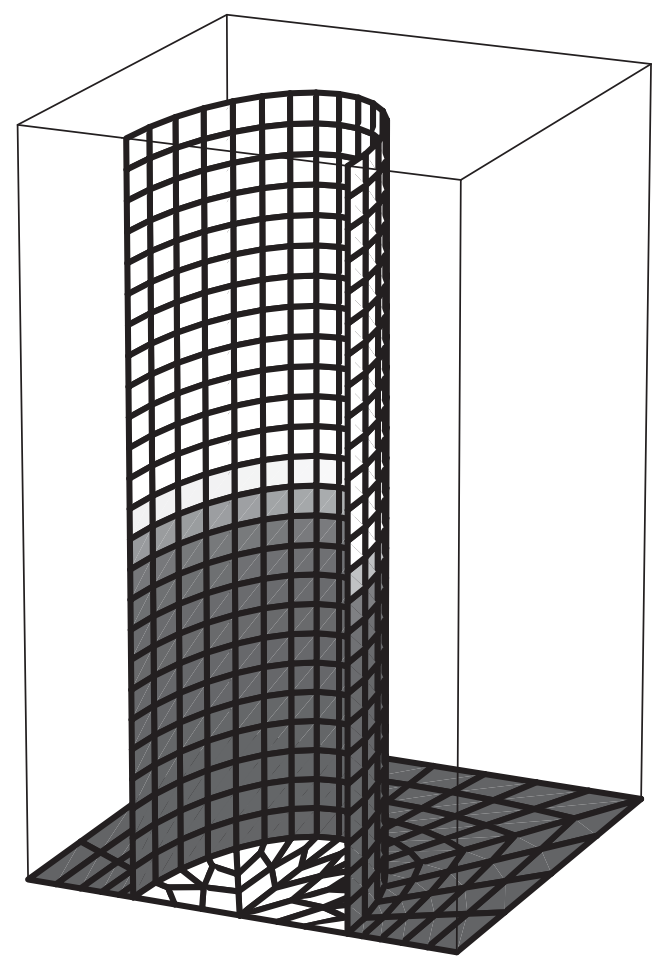

Figure 4. Fibre-reinforced material: representative cell, damaged zones.

the frictional decohesive process, matrix-fibre physical constants, and also fibrefibre, matrix-matrix physical constants at a decohesive zone located at the lower section. In this example, the chosen values allow a progressive decohesive process at the fibre-matrix interface, and a brittle fracture process at the lower section, across the matrix. In this particular case, one observes a crack in the matrix bridged by the fibre: since the fibre-matrix interface is weak, the matrix crack is soon deviated at the fibre-matrix interface (white, $\beta=1$; black, $\beta=0$ ). The fibre, where it becomes unstuck from the matrix, is still extracted with friction. This dissipative process results in a global ductile behaviour of the composite material. More details may be found in Monerie (2000).

\section{An example of a building made of blocks}

In the following example, a numerical test dome, $10 \mathrm{~m}$ in diameter, made of 300 granite elastic blocks, set on 4 pillars, is subjected to gravity. Each block is composed of $8 \mathrm{H} 8$ finite elements. The structure is composed of $2400 \mathrm{H} 8$ elements, 8100 nodes, 24300 degrees of freedom. Each block face has $4 \times 4=16$ candidate points to contact. The total number of candidates to contact is 9176 . The adopted cohesive frictional law is the Mohr-Coulomb law. Figures 5 and 6, from slightly different angles of view, show the bonding with thick joints (actually numerically infinitely thin), and the mesh. The gravity is applied to an unstressed initial configuration. An equilibrium is reached when some highly damped transient state has vanished. In figure 5, blocks are laid without any cohesive properties. One sees that some blocks 


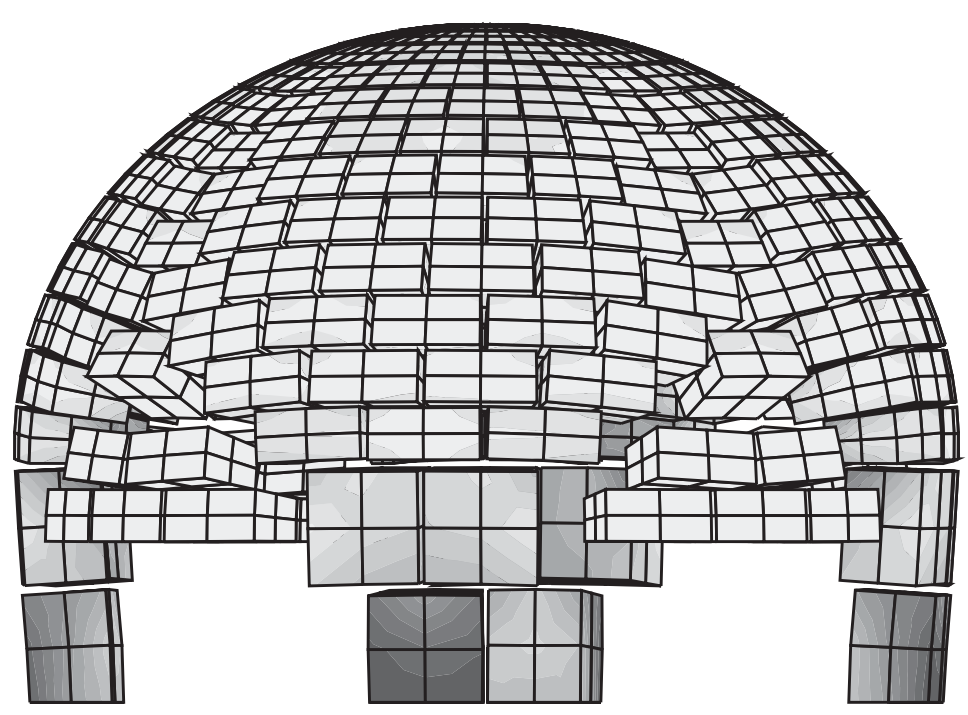

Figure 5. Non-cohesive dome.

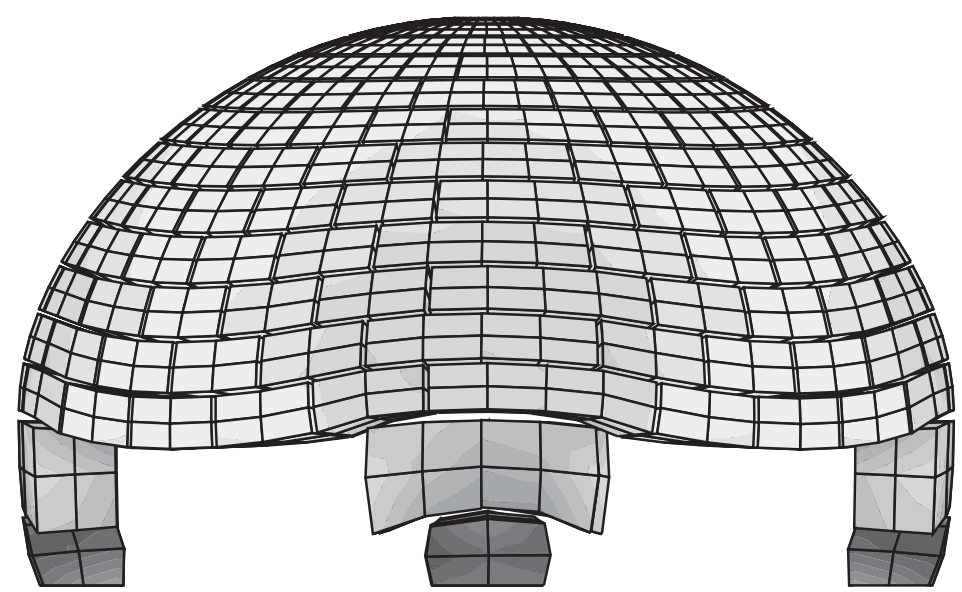

Figure 6. Cohesive dome.

are beginning to fall between pillars (displacements are magnified $2 \times 10^{4}$ times). In the second example, some cohesion has been introduced, as much to allow any joint to resist a tensile force equal to the weight of six blocks, enough to ensure the ability of six-block layers between pillars to resist gravity. In these circumstances the dome is stable (displacements are magnified $3 \times 10^{5}$ times). The scale shows the Von Mises stresses and particularly, compression in the pillars (black) and traction in layers between pillars (white). (See Acary \& Jean (1998, 2000).)

\section{Annex: the two-dimensional 'Signorini, $\mu$-Coulomb, standard' problem solution}

One sets

$$
\mathcal{W}=\left(\begin{array}{ll}
\mathcal{W}_{\mathrm{TT}} & \mathcal{W}_{\mathrm{TN}} \\
\mathcal{W}_{\mathrm{NT}} & \mathcal{W}_{\mathrm{NN}}
\end{array}\right), \quad h \mathcal{R}_{\text {loc stick }}=-\mathcal{W}^{-1} \mathcal{U}_{\text {loc free }}
$$


A shorter notation is adopted, $\left(\mathcal{U}_{\text {free }}=\mathcal{U}_{\text {loc free, }}, \mathcal{R}_{\text {stick }}=\mathcal{R}_{\text {loc stick }}\right)$. It is supposed that $\mathcal{W}$ is positive definite. The term $\mathcal{U}_{\text {free }},\left(\mathcal{U}_{\text {loc free }}\right)$ is the free local velocity, i.e. the local velocity when reaction is discarded on the concerned contact, $\mathcal{R}=0$; the term $\mathcal{R}_{\text {stick }},\left(\mathcal{R}_{\text {loc stick }}\right)$ may be viewed as the reaction when a null relative velocity is imposed, $\mathcal{U}=0$.

$$
\text { It is supposed }-1<-\mu \frac{\mathcal{W}_{\mathrm{NT}}}{\mathcal{W}_{\mathrm{NN}}}<1
$$

The standard solution is

if $\mathcal{U}_{\text {free } \mathrm{N}}>0$, then: no contact,

$h \mathcal{R}=0$

if $\mathcal{U}_{\text {free } \mathrm{N}} \leqslant 0$ and $\mathcal{R}_{\text {stick } \mathrm{T}}+\mu \mathcal{R}_{\text {stick } \mathrm{N}}<0$, then: forward frictional sliding,

$h \mathcal{R}_{\mathrm{T}}=-\mu h \mathcal{R}_{\mathrm{N}}, \quad h \mathcal{R}_{\mathrm{N}}=-\frac{1}{\left(1-\mu\left(\mathcal{W}_{\mathrm{NT}} / \mathcal{W}_{\mathrm{NN}}\right)\right) \mathcal{W}_{\mathrm{NN}}} \mathcal{U}_{\text {free } \mathrm{N}}$

if $\mathcal{U}_{\text {free } \mathrm{N}} \leqslant 0$ and $\mathcal{R}_{\text {stick } \mathrm{T}}-\mu \mathcal{R}_{\text {stick } \mathrm{N}}>0$, then: backward frictional sliding,

$h \mathcal{R}_{\mathrm{T}}=\mu h \mathcal{R}_{\mathrm{N}}, h \mathcal{R}_{\mathrm{N}}=-\frac{1}{\left(1+\mu\left(\mathcal{W}_{\mathrm{NT}} / \mathcal{W}_{\mathrm{NN}}\right)\right) \mathcal{W}_{\mathrm{NN}}} \mathcal{U}_{\text {free } \mathrm{N}}$

if $\mathcal{U}_{\text {free } \mathrm{N}} \leqslant 0$ and $\mathcal{R}_{\text {stick } \mathrm{T}}+\mu \mathcal{R}_{\text {stick } \mathrm{N}} \geqslant 0$ and $\mathcal{R}_{\text {stick } \mathrm{T}}-\mu \mathcal{R}_{\text {stick } \mathrm{N}} \leqslant 0$, then: sticking,

$h \mathcal{R}=h \mathcal{R}_{\text {stick }}$

\section{References}

Acary, V. \& Jean, M. 1998 In Monuments 98, Workshop on Seismic Performances of Monuments, 12-14 November 1998 (ed. Y. Pinto \& M. Géradin), pp. 69-78.

Acary, V. \& Jean, M. 2000 In 5th Int. Conf. on Computational Structures Technology (CST 2000) Leuven, Belgium, 6-8 September 2000 (ed. B. H. V. Topping). Civil-Comp Press.

Alart, P. \& Curnier, A. 1988 A generalized Newton method for contact problems with friction. J. Méc. Théor. Appliq. 7, 67-82.

Alart, P. \& Curnier, A. 1991 A mixed formulation for frictional contact problems prone to Newton-like solution method. Comput. Meth. Appl. Mech. Engng 92, 353-375.

Allix, O., Daudeville, L. \& Ladevèze, P. 1989 In Proc. MECAMAT: Mechanics and Mechanisms of Damage in Composites and Multi-materials, Saint-Etienne, 15-17 Novembre 1989 (ed. D. Baptiste), p. 143.

Allix, O., Ladevèze, P. \& Corigliano, A. 1995 Damage analysis of interlaminar fracture specimens. Composite Struct. 31, 61-74.

Barenblatt, G. I. 1962 The mathematical theory of equilibrium cracks in brittle fracture. Adv. Appl. Mech. 7, 55-129.

Cambou, B. \& Jean, M. 2001 Micromécanique des matériaux granulaires. Hermès.

Cangémi, L., Cocu, M. \& Raous, M. 1996 Adhesion and friction model for the fibre/matrix interface of a composite. In Proc. Third Biennal Joint Conf. on Engineering System Design and Analysis, Montpellier, 1-4 July 1996, pp. 157-163. ASME.

Chaboche, J.-L., Girard, R. \& Levasseur, P. 1997 On the interface debonding models. Int. J. Damage Mech. 6, 220-257.

Chabrand, P., Dubois, F. \& Raous, M. 1995 Programmation mathématique pour le contact avec frottement et comparaison avec d'autres méthode. In Actes du 2ème Colloque National en Calcul des Structures. Hermès. 
Costanzo, F. \& Walton, J. R. 1997 A study of dynamic crack growth in elastic materials using a cohesive zone model. Int. J. Engng Sci. 35, 1085-1114.

De Saxcé, G. \& Feng, Z. Q. 1991 New inequation and functional for contact with friction. J. Mech. Struct. Mach. 19, 301-325.

Dugdale, D. S. 1960 Yielding of steel sheets containing slits. J. Mech. Phys. Solids 8, 100-104.

Frémond, M. 1982 Adhésion et contact unilatéral. In Contact Mechanics and Wear of Rail/Wheel Systems, Vancouver, British Columbia, 6-9 July 1982, pp. 63-77. University of Waterloo Press.

Ida, Y. 1972 Cohesive force across the tip of a longitudinal shear crack and Griffith's specific surface energy. J. Geophys. Res. 77, 3796-3805.

Jean, M. 1995 Frictional contact in rigid or deformable bodies: numerical simulation of geomaterials. Elsevier.

Jean, M. 1999 In Computer Meth. Appl. Mech. and Engng. Special Issues on Computational Modeling on Contact and Friction (ed. J. A. C. Martins \& A. Klarbring), vol. 177, pp. 235257.

Jean, M. \& Moreau, J.-J. 1992 In Proc. Contact Mech. Int. Symp. (ed. A. Curnier), pp. 31-48.

Klarbring, A. 1990 Derivation and analysis of rate boundary-value problems of frictional contact. Eur. J. Mech. A 9, 953-985.

Michel, J.-C. \& Suquet, P. 1994 An analytical and numerical study of the overall behaviour of metal-matrix composites. Model. Simul. Mater. Sci. Engng 2, 637-658.

Monerie, Y. 2000 Fissuration des matériaux composites: rôle de l'interface fibre/matrice. PhD thesis, Université de la Méditerranée.

Moreau, J.-J. 1994 Some numerical methods in multibody dynamics: application to granular materials. Eur. J. Mech. A 13, 93-114.

Moreau, J.-J. 1999 In Unilateral multibody dynamics (ed. F. Pfeiffer \& C. Glocker). Dordrecht: Kluwer.

Needleman, A. 1987 A continuum model for void nucleation by inclusion debonding. J. Appl. Mech. 54, 525-531.

Needleman, A. 1990 An analysis of tensile decohesion along an interface. J. Mech. Phys. Solids 38, 289-324.

Needleman, A. 1992 Micromechanical modeling of interfacial decohesion. Ultramicroscopy 40, 203-214.

Needleman, A. \& Rosakis, A. J. 1999 The effect of bond strenght and loading rate on the conditions governing the attainment of intersonic crack growth along interfaces. J. Mech. Phys. Solids 47, 2411-2450.

Palmer, A. C. \& Rice, J. R. 1973 The growth of slip surfaces in the progressive failure of overconsolidated clay. Proc. R. Soc. Lond. A 332, 527-548.

Raous, M., Cangémi, L. \& Cocu, M. 1997 Un modèle couplant adhérence et frottement pour le contact unilatéral entre deux solides déformables. C. R. Acad. Sci. Paris Sér. IIb 329, 503-509.

Raous, M., Cangémi, L. \& Cocu, M. 1999 Consistent model coupling adhesion, friction and unilateral contact. Comput. Meth. Appl. Mech. Engng 177, 383-399.

Rice, J. R. 1968 In Fracture (ed. H. Liebowitz), vol. 2, pp. 191-311. Academic.

Rose, J. H., Ferrante, J. \& Smith, J. R. 1981 Universal binding energy curves for metals and bimetallic interfaces. Phys. Rev. Lett. 47, 675-678.

Rose, J. H., Smith, J. R. \& Ferrante, J. 1983 Universal features of bonding in metals. Phys. Rev. B 28, 1835-1845.

Siegmund, T. \& Needleman, A. 1997 A numerical study of dynamic crack growth in elasticviscoplastic solids. Int. J. Solids Struct. 34, 769-787.

Siegmund, T., Fleck, N. A. \& Needleman, A. 1997 Dynamic crack growth across an interface. Int. J. Fracture 85, 381-402. 
Tvergaard, V. 1990 Effect of fibre debonding in a whisker-reinforced metal. Mater. Sci. Engng A 125, 203-213.

Tvergaard, V. 1991 Effect of thermally induced residual stresses on the failure of a whiskerreinforced metal. Mech. Mater. 11, 149-161.

Tvergaard, V. \& Hutchinson, J. W. 1992 The relation between crack growth resistance and fracture process parameters in elastic-plastic solids. J. Mech. Phys. Solids 40, 1377-1397.

Vola, D., Pratt, E., Jean, M. \& Raous, M. 1998 Consistent time discretization for dynamical frictional contact problems and complementarity techniques. Rev. Eur. Eléments Finis 7, $149-162$.

Xu, X.-P. \& Needleman, A. 1994 Numerical simulations of fast crack growth in brittle solids. $J$. Mech. Phys. Solids 42, 1397-1434.

Xu, X.-P., Needleman, A. \& Abraham, F. F. 1997 Effect of inhomogeneities on dynamic crack growth in an elastic solid. Model. Simul. Mater. Sci. Engng 5, 489-516.

Xu, G., Bower, A. F. \& Ortiz, M. 1998 The influence of crack trapping on the toughness of fiber reinforced composites. J. Mech. Phys. Solids 46, 1815-1833. 